\title{
Accuracy of fluoroscopic-guided genicular nerve blockade: a need for revisiting anatomical landmarks
}

\author{
Loïc Fonkoue, ${ }^{1,2}$ Catherine Wydemans Behets, ${ }^{1}$ Arnaud Steyaert, ${ }^{3,4}$ \\ Jean-Eric Kouame Kouassi, ${ }^{2}$ Christine Detrembleur, ${ }^{2}$ Bernard Le Polain De Waroux, ${ }^{3}$ \\ Olivier Cornu ${ }^{2,5}$
}

\begin{abstract}
'Department of Morphology, Experimental and Clinical Research Institute, Universite catholique de Louvain, Brussels, Belgium

${ }^{2}$ Neuro-Musculo-Skeletal Department (NMSK).

Experimental and Clinical Research Institute, Université catholique de Louvain, Brussels, Belgium

${ }^{3}$ Department of Anesthesia and Pain Medicine, Cliniques universitaires Saint-Luc, Bruxelles, Belgium ${ }^{4}$ Institute of Neuroscience (IONS), Universite catholique de Louvain, Brussels, Belgium ${ }^{5}$ Department of Orthopedics and Trauma, Cliniques universitaires Saint-Luc, Bruxelles, Belgium
\end{abstract}

\section{Correspondence to} Dr Loïc Fonkoue, Department of Morphology, Experimental and clinical research institute, Universite catholique de Louvain, Brussels 1348, Belgium; loic.fonkoue@uclouvain.be

Received 5 February 2019 Revised 23 July 2019 Accepted 12 August 2019 Published Online First 26 August 2019

\section{ABSTRACT}

Background and objectives Genicular nerve blockade (GNB) and radiofrequency ablation (RFA) have recently emerged as treatment options for patients with chronic knee pain. However, an increasing number of anatomical studies and systematic reviews concluded that the anatomical basis for needle placement was unclear, incomplete and somewhat inaccurate. This study was designed to assess the accuracy of updated anatomical landmarks for fluoroscopy-guided blockade of the consistent genicular nerves in a cadaveric model. Methods Based on a comprehensive review of recent anatomical studies and prior dissection of 21 fresh cadaver knees, we defined bony landmarks with high likelihood of successful ablation of the five consistent genicular nerves (GN). We tested the accuracy of GNBs using the above-stated anatomical landmarks in 10 intact fresh cadaveric knees. Needle placement was guided by fluoroscopy and $0.5 \mathrm{~mL}$ of $0.1 \%$ methylene blue was injected at the site of each nerve. The knees were subsequently dissected to assess the accuracy of the injections. If the nerve was dyed with blue ink, the placement was considered accurate.

Results The accuracy of our injections was 100\% for the superior medial genicular nerve, inferior medial GN, infrapatellar branch of saphenous nerve and recurrent fibular nerve. The superior lateral GN was dyed in $90 \%$ of specimens.

Conclusion This study provides physicians with precise anatomical landmarks for the five consistent GN for fluoroscopic-guided GNB. Our revised technique, which targets more nerves with increased accuracy, could potentially lead to improved therapeutic benefits on chronic knee pain.

\section{INTRODUCTION}

Chronic knee pain conditions are very frequent in elderly, mainly due to osteoarthritis (OA). Their treatment remains a great challenge among medical practitioners. Conservative therapies have shown limited efficacy regarding long-term results. ${ }^{1}$ While total knee arthroplasty (TKA) remains the gold standard treatment for advanced stages of knee OA, up to $44 \%$ of patients report persistent pain after TKA, with $15 \%$ suffering from extremely severe pain. ${ }^{23}$

Genicular nerve block (GNB) and radiofrequency ablation (RFA) have recently emerged as novel alternatives in the treatment of chronic knee pain. ${ }^{4-6}$ These methods are based on the principle that interrupting sensory nerve fibers to a painful structure may alleviate pain and restore function. Their targets are sensory nerves which lie on the periosteum before entering the knee joint capsule and can easily be located using bony landmarks under fluoroscopic guidance. ${ }^{48}$ The success of GNB and RFA depends on the target nerve being included within the anaesthetized or thermocoagulated tissue volume. ${ }^{7}$ It requires precise localization of the articular branches innervating the knee joint capsule, given the limited size of RFA lesions. The majority of the clinical studies involve fluoroscopic-guided $\mathrm{RFA}^{9-14}$ following Choi et al's ${ }^{4}$ original landmarks, even though recent anatomical studies have shown that their anatomic foundations were somewhat inaccurate or incomplete..$^{5}$ 15-18 This may explain the variable results of these studies in terms of intensity and duration of the pain relief. Optimizing the effectiveness of the technique relies on lesioning more genicular nerves, in an accurate and safe manner. ${ }^{18}$

To achieve this aim, a comprehensive review of recent anatomical studies ${ }^{5}$ 15-20 $^{2}$ and subsequent anatomical dissection of 21 fresh cadaveric knees allowed us to identify five consistent and easily targetable articular branches innervating the knee joint capsule: the superior medial, superior lateral, inferior medial genicular nerves, the recurrent fibular nerve and the infrapatellar branch of the saphenous nerve. Their precise clinical and radiological landmarks with high likelihood of ablation success were refined. This experimental study sought to assess the accuracy of these new anatomical landmarks for fluoroscopic-guided GNB in cadaveric models, and define a potentially more effective treatment approach targeting more genicular nerves than Choi et al. ${ }^{4}$ To our knowledge, no previous cadaveric study assessing the accuracy of needle placement under fluoroscopic guidance for GNB/RFA is found in the literature.

\section{METHODS}

This study was conducted at the laboratories of anatomy and experimental surgery of the Université catholique de Louvain. It followed the anatomical dissection of 21 cadaver knees by the same investigators, who intended to determine different patterns of sensory innervation of the knee, select consistently targetable genicular nerves and determine their anatomical landmarks for therapeutic purpose. ${ }^{21}$ Here, we tested the accuracy of the revisited anatomical landmarks for GNB in 10 knees (five left and five right) with physical integrity, from 

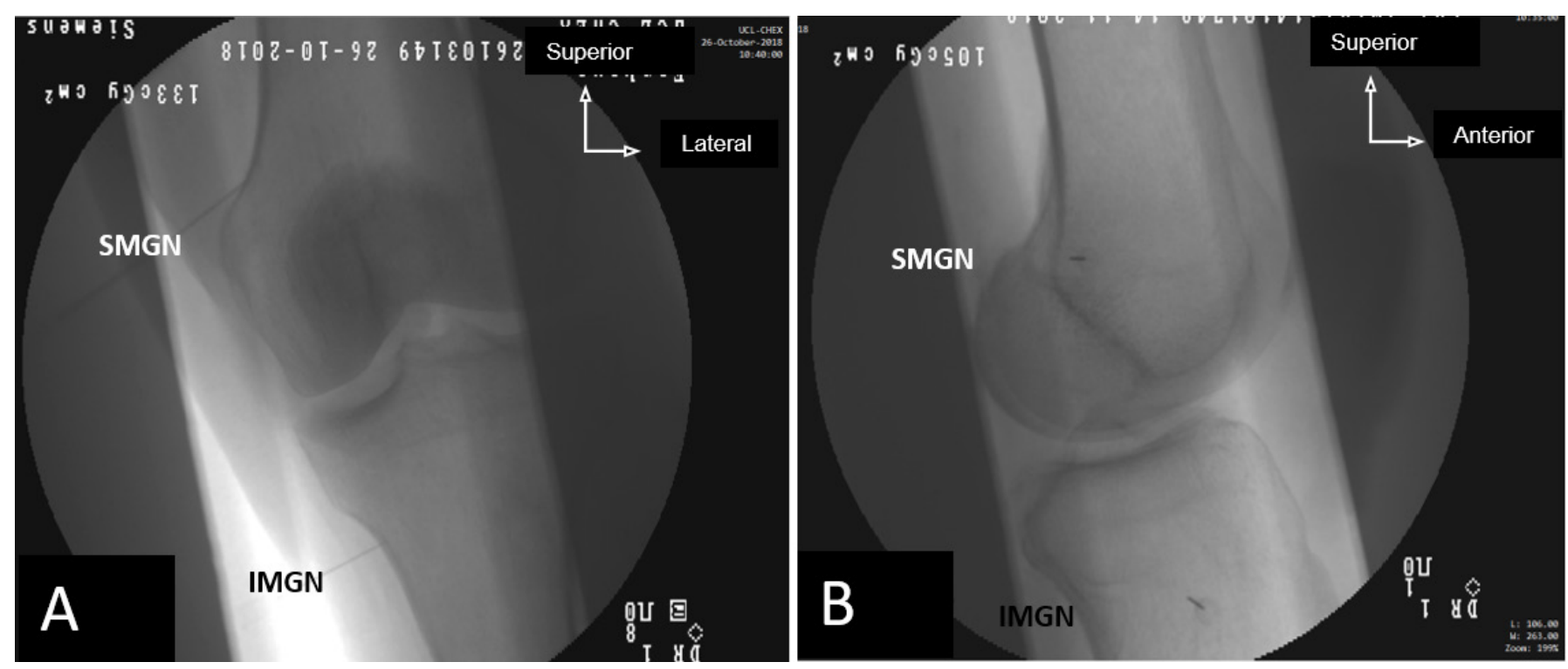

Figure 1 Fluoroscopic images of the final needle position for the injection of the SMGN and the IMGN. (A) Anterior-posterior view and (B) lateral view. IMGN, inferior medial genicular nerve; SMGN, superior medial genicular nerve.

10 fresh cadavers, by injecting $0.5 \mathrm{~mL}$ of methylene blue after fluoroscopy-assisted needle placement, as it would customarily be done in a pain clinic. The cadavers, five male and five female, aged $89.6 \pm 5.8$ years, with no history of surgery or major procedure in their knees, were donated to the laboratory of anatomy in accordance with Belgium law and regulations.

\section{Injection procedure}

The knees were injected by an experienced physician who specializes in local infiltration techniques in our institution, following the procedures and the revisited anatomical landmarks described below. Each injection was performed after the correct needle placement targeting specific anatomical landmark under fluoroscopic guidance, as it would be done for GNB. We targeted five articular nerves supplying the knee joint capsule, whose anatomical landmarks around the knee were found to be consistent.

\section{The superior medial genicular nerve (SMGN)}

Under fluoroscopic guidance, in supine position with the knee in extension, a 22-gage needle was inserted toward the area just anterior to the adductor tubercle (AT). On the anterior-posterior (A-P) view, the needle tip was advanced to the superior edge of the medial femoral condyle until it touched the bone (figure $1 \mathrm{~A}$ ). Then the $\mathrm{C}$-arm was rotated to have a true lateral view of the knee with alignment of the two femoral condyles. The needle position was then readjusted to fit just anterior to the junction between medial condyle and posterior femoral cortex (figure 1B), but not at the middle of the femoral shaft like currently done in clinical practice. ${ }^{411-13}$ After verification of correct placement of the needle tip, $0.5 \mathrm{~mL}$ of $0.1 \%$ methylene blue was injected and the needle was removed.

\section{The inferior medial genicular nerve (IMGN)}

The needle placement location for the IMGN was like currently done in clinical practice. ${ }^{4}$

\section{The superior lateral genicular nerve (SLGN)}

In supine position with the knee in extension, the C-arm was positioned to have a true lateral view. The needle was inserted toward the target area located at the junction between superior edge of the lateral femoral condyle and the posterior femoral cortex, until the tip touched the bone (figure 2A). Then, the needle was withdrawn for about $2 \mathrm{~mm}$. No readjustment was needed in A-P view; this point corresponds to the upper edge of the lateral condyle height (figure $2 \mathrm{~B}$ ). Injection of $0.5 \mathrm{~mL}$ of $0.1 \%$ methylene blue was performed and the needle was removed.

\section{The recurrent fibular nerve (RFN)}

In the supine position with the leg in full extension, we first identified the Gerdy's tubercle and the tibial tuberosity by simple palpation. A longitudinal line was drawn below the Gerdy's tubercle and the target point was located on this line, $1 \mathrm{~cm}$ below the inferior edge of the tubercle (figure $3 \mathrm{~A}$ ). The needle was inserted at this point and advanced until the tip touched the bone. We performed the injection of $0.5 \mathrm{~mL}$ of $0.1 \%$ methylene blue with the needle tip still in contact with the bone, before removal of the needle.

\section{The infrapatellar branch of saphenous nerve (IPBSN)}

A target line was adapted from Hu's description, ${ }^{20}$ slightly modified according to our prior cadaveric dissections. We drew a transversal line passing by the apex patellae and another one passing by the top of the tibial tuberosity. Four centimeters medially to the apex patellae, we drew a longitudinal line connecting the two transversal lines (figure 3B). This longitudinal line corresponds to the targeted treatment line found to contain $95 \%$ of nerve branches in our prior cadaveric dissection of 21 specimens, rather than $85 \%$ for Hu's line. ${ }^{20}$ We injected $1.0 \mathrm{~mL}$ of $0.1 \%$ methylene blue deeply under the subcutaneous tissue, throughout this target longitudinal line.

\section{Assessment}

After completion of all the injections in the 10 specimens, the limbs were dissected to evaluate the accuracy of the injections, based on location of the blue ink in the tissues. The dissections were performed by experienced anatomists and consultant orthopedic surgeon with more than 8 years of experience in cadaveric dissection, who also conducted previous cadaveric study on the 

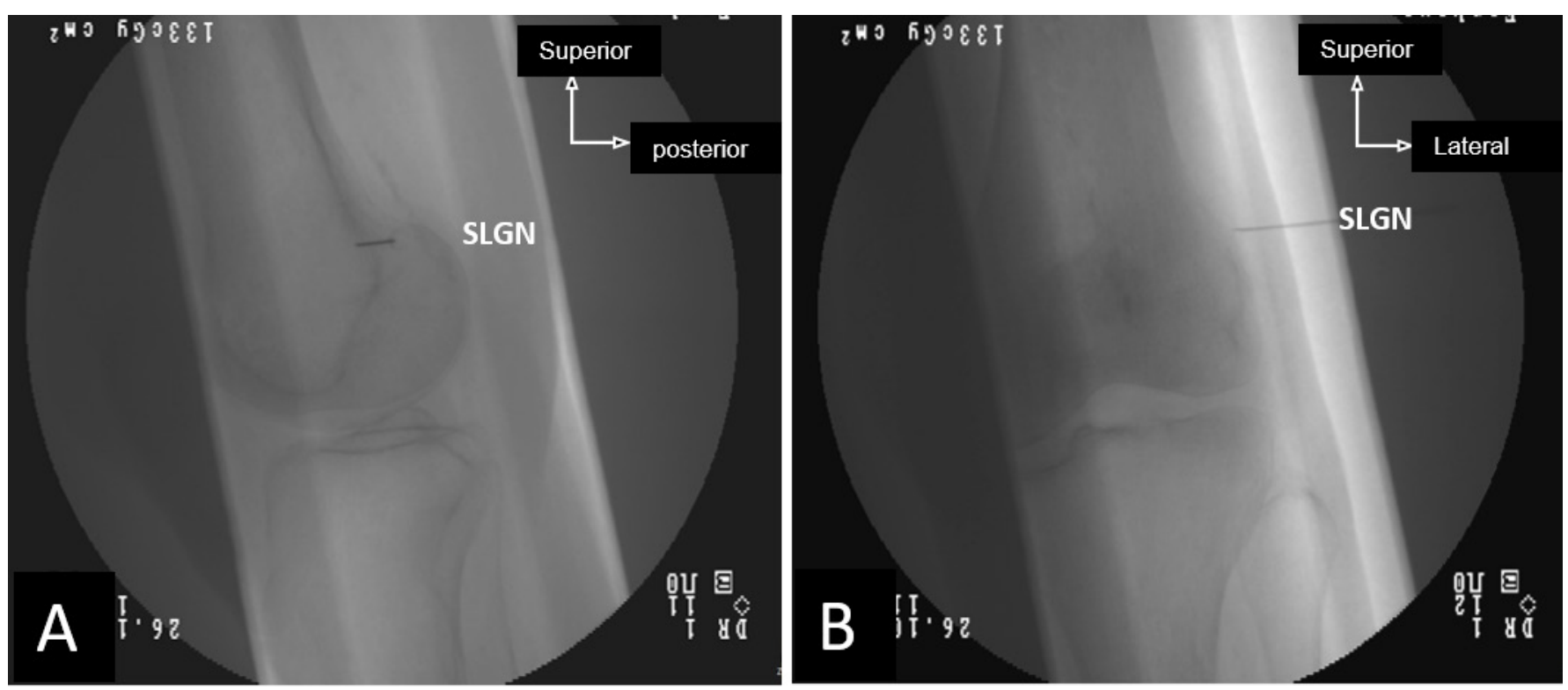

Figure 2 Fluoroscopic images of the final needle position for the injection of the SLGN. (A) Lateral view and (B) anterior-posterior view. SLGN, superior lateral genicular nerve.

knee nerve supply. Each injection was considered accurate only if the nerve was dyed with the blue color during dissection. All other locations were considered inaccurate. We also looked for the safety of the injections. Injection was considered safe if no major nerve trunk (sciatic, tibial, common fibular or saphenous
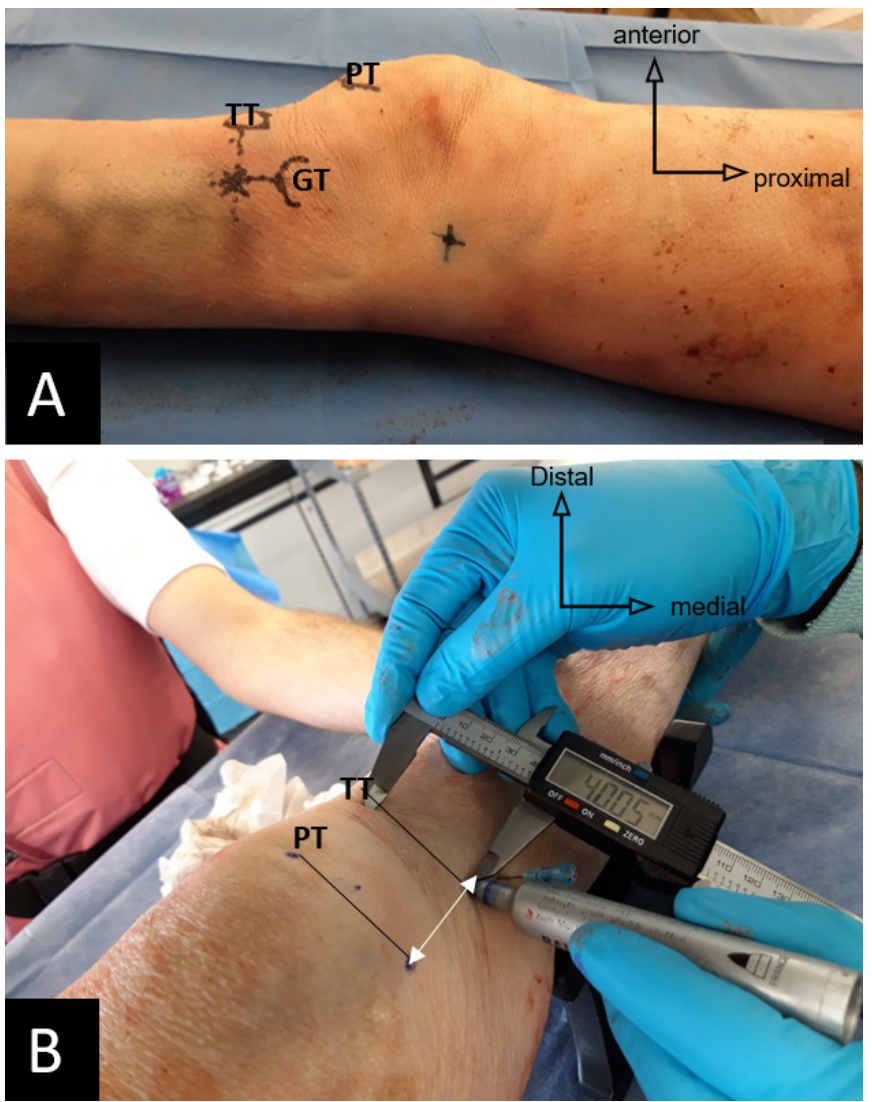

Figure 3 (A) Anatomical landmarks for the RFN. (B) Treatment line for the IPBSN (white double arrow). GT, Gerdy's tubercle; IPBSN, infrapatellar branch of saphenous nerve; P, patella; PT patellar tip (or apex patellae); TT, tibial tuberosity; RFN, recurrent fibular nerve nerves) or major vessels were found dyed during dissection. The results were compiled for the analysis of the accuracy and the safety of the injections with these revised anatomical landmarks.

\section{RESULTS}

The postinjection cadaveric dissections revealed that $100 \%$ of the needle placement for the SMGN, IMGN, RFN, IBPSN, and $90 \%$ for the SLGN were accurate. Concerning the safety, no major nerve trunk or major vessel was found dyed with blue ink during dissection.

In the 10 specimens, the SMGN was found, dyed with blue, in front of the AT (figure 4). It was clearly a direct articular branch from the nerve to the vastus medialis in all the specimens, descending in a tunnel through the fascia of the vastus medialis muscle, then running on the adductor magnus tendon toward the AT. Blue coloring agent was spread $1-2 \mathrm{~cm}$ around the AT, and no nerve trunk (motor branches of the nerve to vastus medialis, saphenous nerve) was dyed.

The IMGN was found dyed with blue in all the specimens, beneath the deep surface of the medial collateral ligament (MCL) of the knee, running toward the inferior-medial quadrant of the anterior knee joint capsule, accompanied with inferior medial genicular vessels (figure 5). The blue ink was spread under the MCL and no other structure was found dyed.

The IPBSN crossed the treatment line in all the 10 specimens, regardless of the level where it detached from the saphenous nerve (figure 6). In three of the 10 cases, we found the IPBSN originating proximally, above the adductor canal and then, running outside it (figure 6-C). However, despite this anatomical variation in their proximal trajectory, their distal portion still crossed the treatment line, so was dyed with blue ink.

The distal portion of the RFN was dyed with blue in all the specimens, just before it reached the inferior lateral aspect of the anterior knee joint capsule (figure 7). This nerve originated from the common fibular nerve and joined the anterior tibial recurrent artery; they ascended together on the periosteum towards the knee joint, passing below the Gerdy's tubercle. Blue ink was localized at the deep surface of the initial fibers of anterior tibial muscle, just below the Gerdy's tubercle. The common fibular 

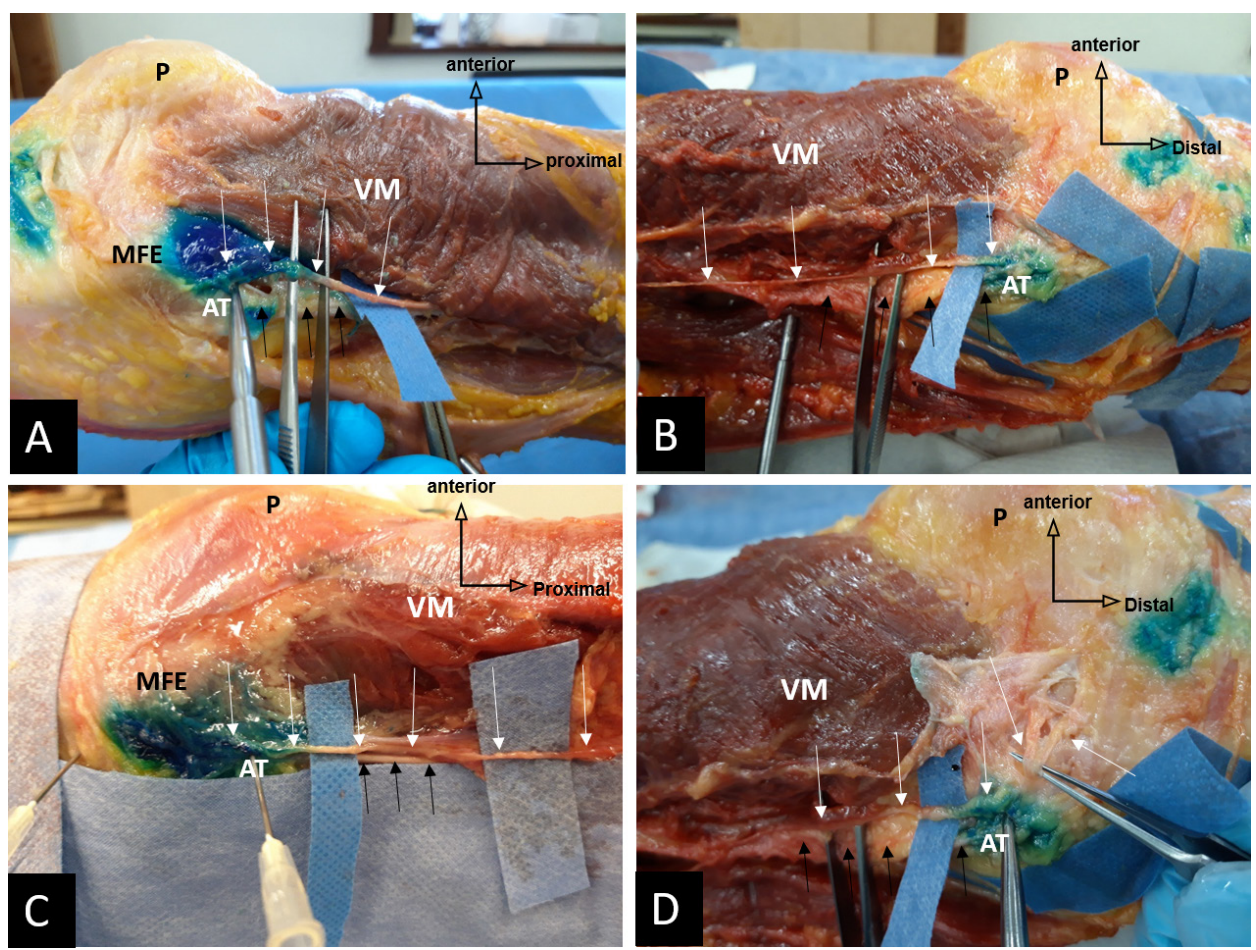

Figure 4 Postinjection anatomical dissection, showing the superior medial genicular nerve (white arrows) in various specimens, running on the adductor Magnus tendon (black arrows), dyed with blue around the AT. See the nerve passing just in front of the AT in all the specimens and accurately targeted. AT, adductor tubercle; MFE, medial femoral epicondyle; P, patella; VM, vastus medialis muscle.

nerve was $4-5 \mathrm{~cm}$ far away from the colored tissue area, and none of the $10 \mathrm{CFN}$ was dyed with blue ink (figure 7).

The SLGN was dyed with blue color in 9 of the 10 specimens (figure 8). It originated from the sciatic nerve in all the specimens, ran under the deep surface of the biceps femoris muscle toward area connecting the lateral femoral condyle and the posterior edge of the lateral side of femoral shaft, and then divided into two endings branches: a transversal branch running forward to the lateral retinaculum (lateral retinacular nerve) and a longitudinal branch descending toward the femorotibial space. The blue ink was localized to this area, dying the SLGN just before its bifurcation. No major nerve trunk (CFN, tibial nerve) was found dyed during dissection. In one of the 10 specimens, the bifurcation of the SLGN was higher, and none of the two branches of the nerve was dyed.

\section{DISCUSSION}

To the best of our knowledge, this is the first study specifically designed to assess the accuracy of anatomical landmarks for fluoroscopic-guided GNB in a cadaveric model. It is based on the anatomically updated data regarding the innervation of the knee joint, which warrants revisiting the widely used technique for fluoroscopic-guided genicular denervation of Choi et al. ${ }^{4} \mathrm{~A}$
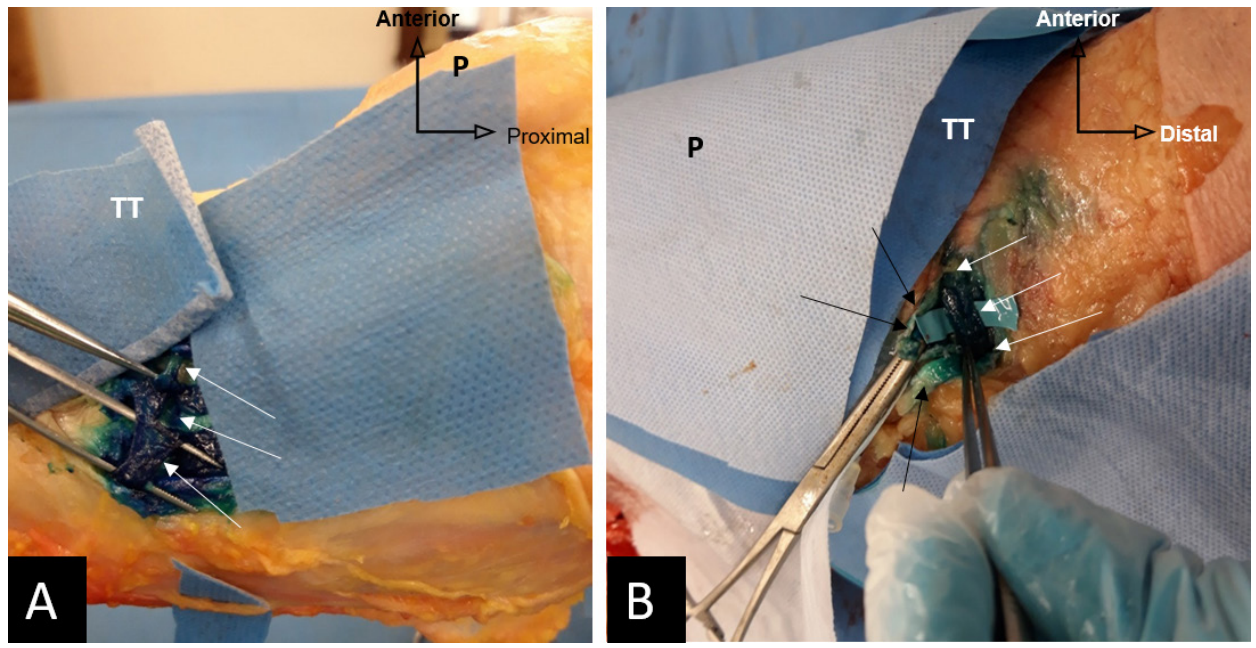

Figure 5 Postinjection anatomical dissection, inferior medial genicular nerve (white arrows) in the bundle with inferior medial genicular artery, dyed in blue, running beneath the medial collateral ligament of the knee (black arrows). (A) Anterior-medial view of a right knee. (B) Anterior-medial view of a left knee. P, patella; TT, tibial tuberosity. 

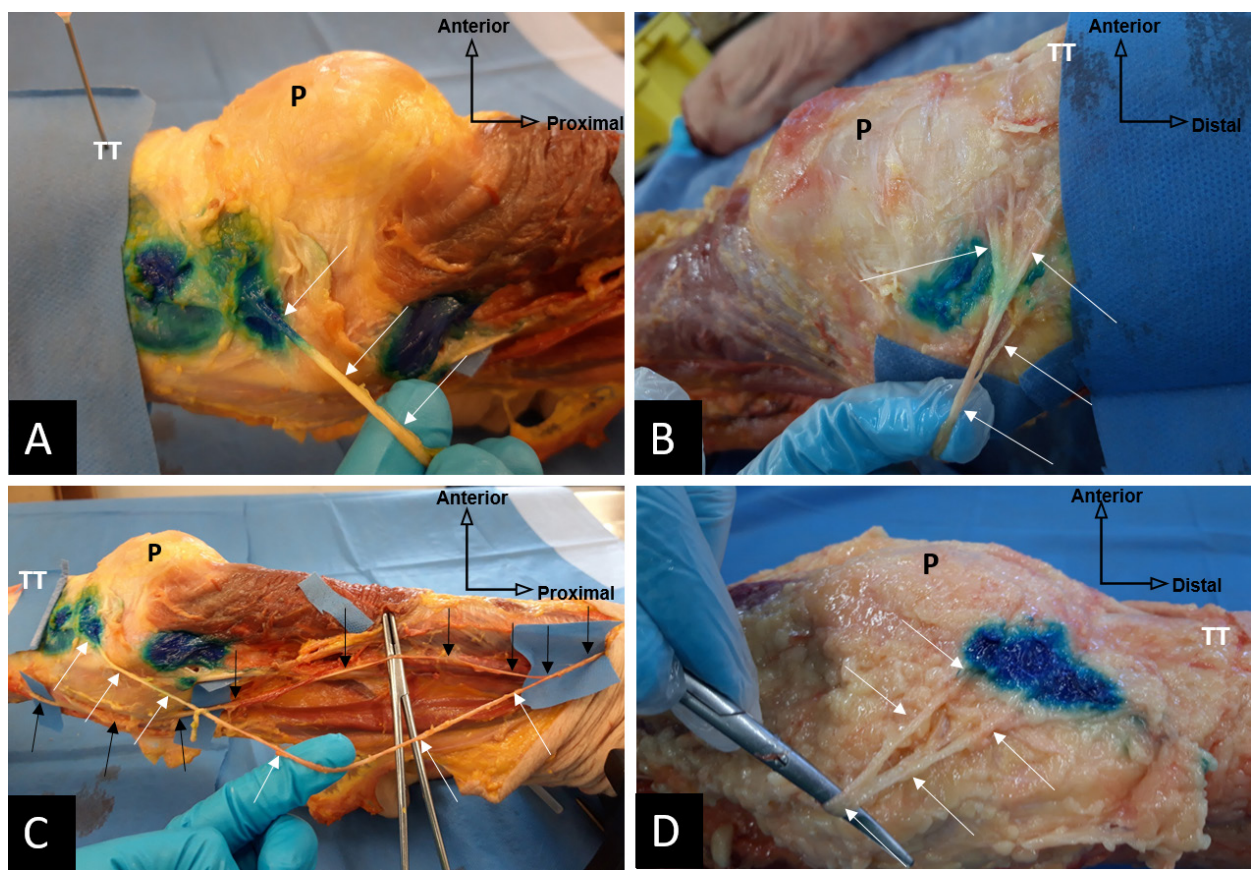

Figure 6 Postinjection anatomical dissection, IPBSN (white arrows) dyed in blue on the treatment line in all the specimens, supplying the inferior medial quadrant of the knee. (A) Anterior-medial view of a right knee, IPBSN crossing the treatment line. (B) Anterior-medial view of a left knee, terminal branches of the IPBSN penetrating the knee capsule, dyed with blue. (C) Anatomical variation of the origin of the IPBSN (white arrows) detached from saphenous nerve (black arrows) proximally above the adductor canal (vasto-adductor membrane removed), but the distal course at the level of the knee remains consistent and crosses the treatment line. (D) Bifurcation of IPBSN, all the branches are included in the tissue volume dyed with blue ink. IPBSN, infrapatellar branch of the saphenous nerve; P, patella; TT, tibial tuberosity.
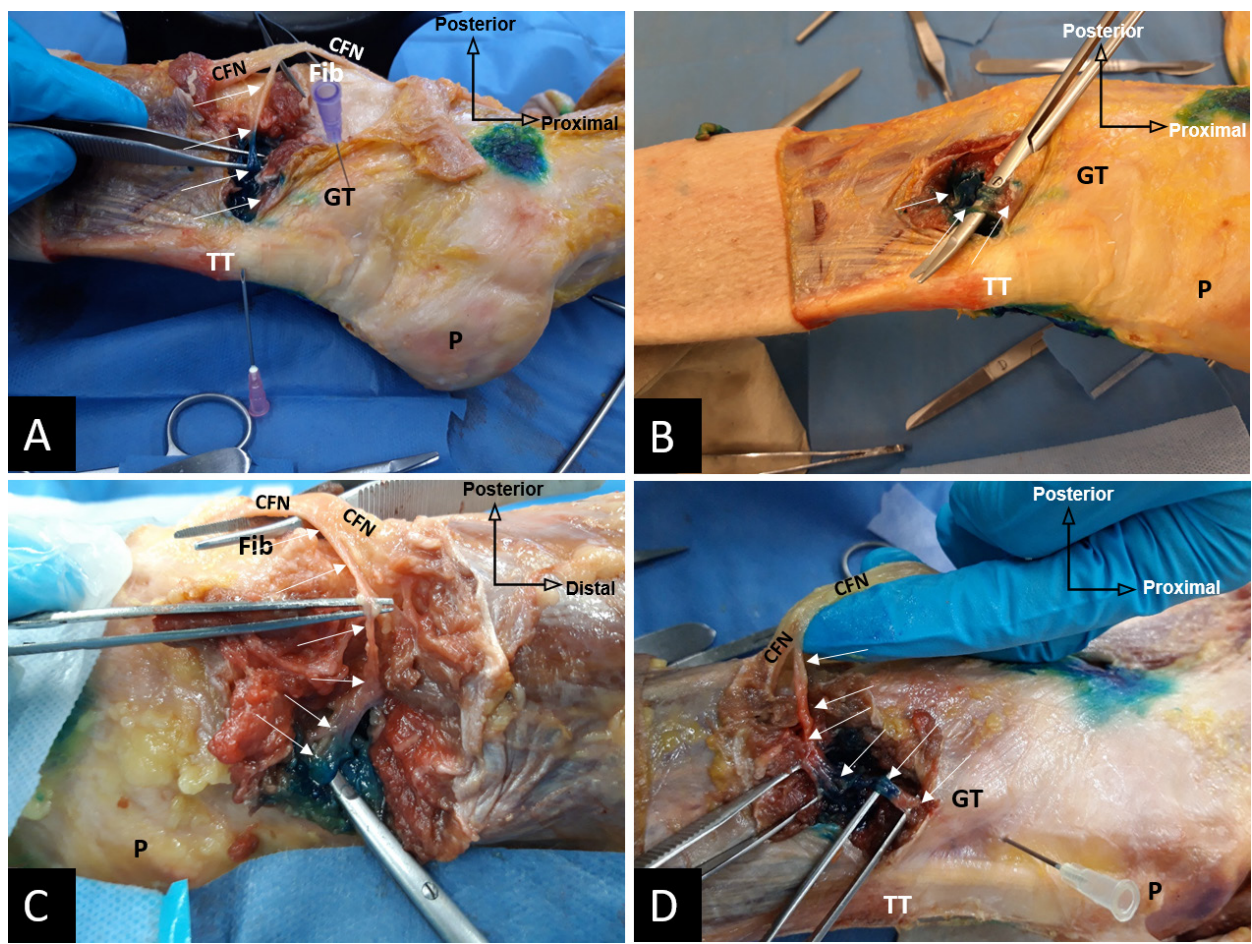

Figure 7 Postinjection anatomical dissection. RFN (white arrows) originating from CFN, joining the anterior tibial recurrent artery at the deep surface of the muscles, and ascending together on the periosteum to supply the inferior lateral quadrant of the knee. (A-D) RFN dyed in blue by deep injection below the GT in various specimens; note the integrity of CFN, which is far away from the colored tissue volume. CFN, common fibular nerve; Fib, fibula head; GT, Gerdy's tubercle; P, patella; RFN, recurrent fibular nerve. 

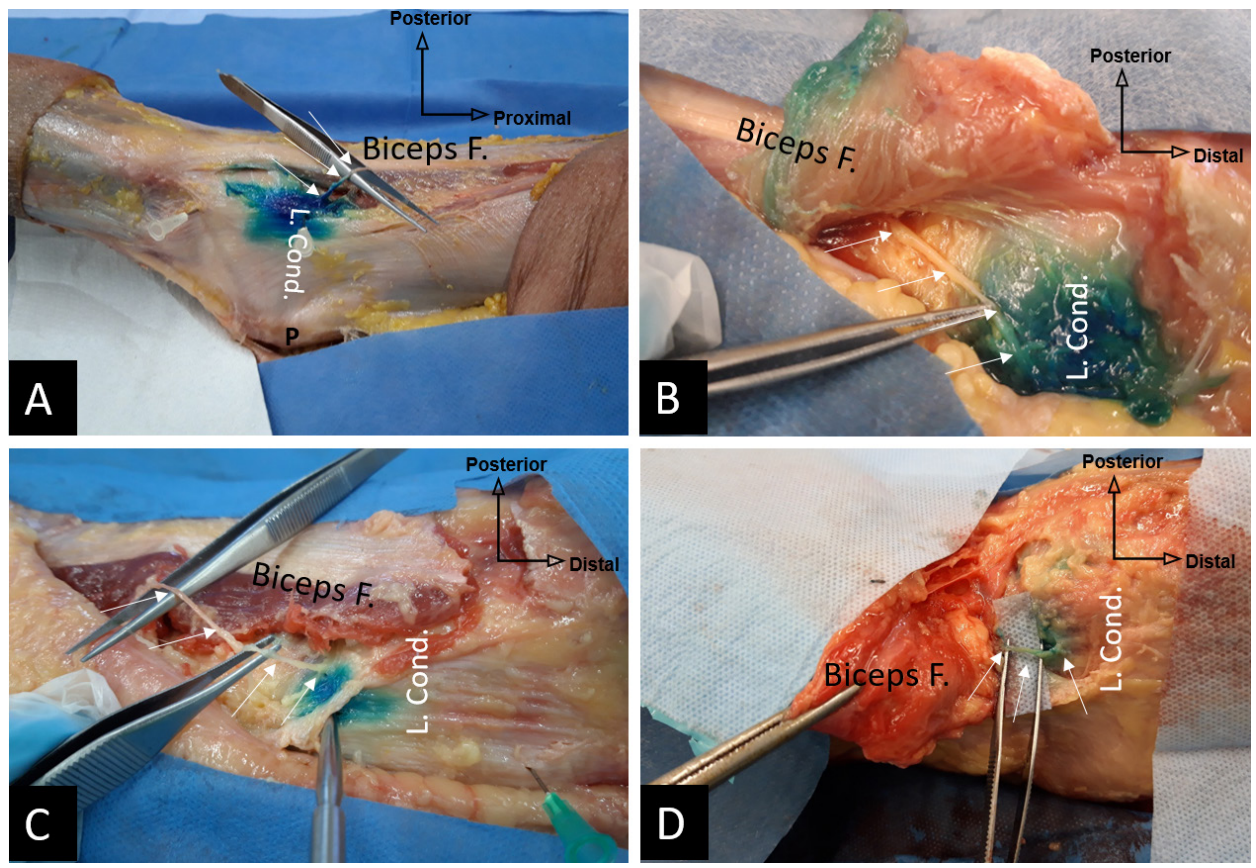

Figure 8 Postinjection anatomical dissection. SLGN (white arrows) from sciatic nerve, descending in an oblique trajectory at the deep surface of the biceps femoris muscle (Biceps F.), toward the area connecting the posterior cortex of the lateral side of the femur shaft and the lateral condyle, before distributing to the superior lateral quadrant of the knee capsule. (A) Posterior-superior-lateral view of a right knee, biceps femoris just retracted. (B-D) Posterior-superior-lateral views of various left knees, biceps femoris sectioned and reflected to highlight the SLGN (white arrows). L. Cond. lateral condyle; Biceps F., biceps femoris; P, patella; SLGN, superior lateral genicular nerve.

recent review concluded that the anatomical basis for radiofrequency cannula placement was unclear ${ }^{22}$ and most of the studies describe lesioning of only three nerves (SMGN, SLGN, and IMGN). This cadaveric study found that our updated anatomical landmarks for the five consistent articular nerves supplying the knee capsule (SMGN, IMGN, SLGN, RFN, and IPBSN) are accurate, safe, and reproducible. To optimize the therapeutic effect of the GNB, we suggest the five-sites denervation technique provided in this study.

There is a lack of consensus concerning the origin and the landmarks of the SMGN. In all our specimens, it was a branch from the nerve to vastus medialis (NVM) and not from tibial nerve like previously suggested by Choi et al. ${ }^{4}$ Tran et al ${ }^{18}$ suggested that it is a branch of femoral nerve, but when looking carefully at the figure $4 \mathrm{C}$ they provided to illustrate this statement, the SMGN is detached from NVM. In the classic fluoroscopic-guided RFA procedure, the SMGN site is located at the confluence of the medial femoral shaft and the medial femoral condyle in the A-P view and at the midpoint of the femoral shaft width in the lateral view. ${ }^{41213}$ We found this landmark inaccurate. Surprisingly, there is a huge discrepancy between anatomical descriptions of the SMGN located by almost all the authors at the $\mathrm{AT}^{5} 151618$ and the targeted point during fluoroscopic-guided RFA. In fact, in the lateral plane radiographic image, the AT is projected at the posterior edge of the area connecting the shaft of the femur and medial condyle. The SMGN should thus be targeted just few millimeters anterior to the AT and not at the midpoint of the femur shaft width (figure 9). In our study, the injection performed just anterior to the AT provided $100 \%$ accuracy on the SMGN.

The classical landmark for the SLGN during RFA procedure is located at the confluence of the femoral shaft and the lateral femoral condyle in the A-P view and the midpoint of the femur A-P width in the lateral view. ${ }^{4} 12$ We found this landmark somewhat inappropriate (figure 9). In this study, on the true lateral view, the injection performed in the area connecting the posterior cortex of the femur shaft and the superior edge of the lateral condyle provided a $90 \%$ success rate on the SLGN. At this level, the needle should not be advanced too far, behind the femur, to avoid spreading to the CFN. We found that the classical landmark corresponds to the trajectory of superior lateral genicular artery (SLGA), which was inconsistently joined by the transversal division from the SLGN (the lateral retinacular nerve) contrary to Sutaria et $a l^{19}$ who found the lateral retinacular nerve (LRN) and SLGA running together in all their specimens. Our landmarks allows us to capture the SLGN before or just at the level of its bifurcation so that all the endings branches were treated in $90 \%$ of specimens. Some authors suggested to target the articular branch from the nerve tovastus lateralis (NVL), ${ }^{7}$ but we found that the higher variability of the distal distribution of the NVL does not allow its precise localization for RFA.

This study is the first to describe a new approach for safe targeting of the RFN without lesioning of the CFN. The RFN plays an important role in sensory innervation of the inferior-lateral quadrant of the knee. ${ }^{7118}$ However, all the authors have excluded it as a potential target for ablation therapy, due to its close proximity to the CFN. This is because they all assumed a target area located on the fibular neck where the RFN originate, which could indeed be dangerous for the CFN trunk. We considered a different approach: targeting the nerve at its distal end, far away from the CFN, just before it reaches the articular capsule. We found that this nerve dives under the deep surface of the muscles to join the anterior tibial recurrent artery at the anterior aspect of the lateral tibial condyle. They ascend together in the same bundle, lying on the periosteum, passing about $1 \mathrm{~cm}$ below the Gerdy's tubercle, and end in the anterolateral knee capsule. The injection performed $1 \mathrm{~cm}$ below the Gerdy's 

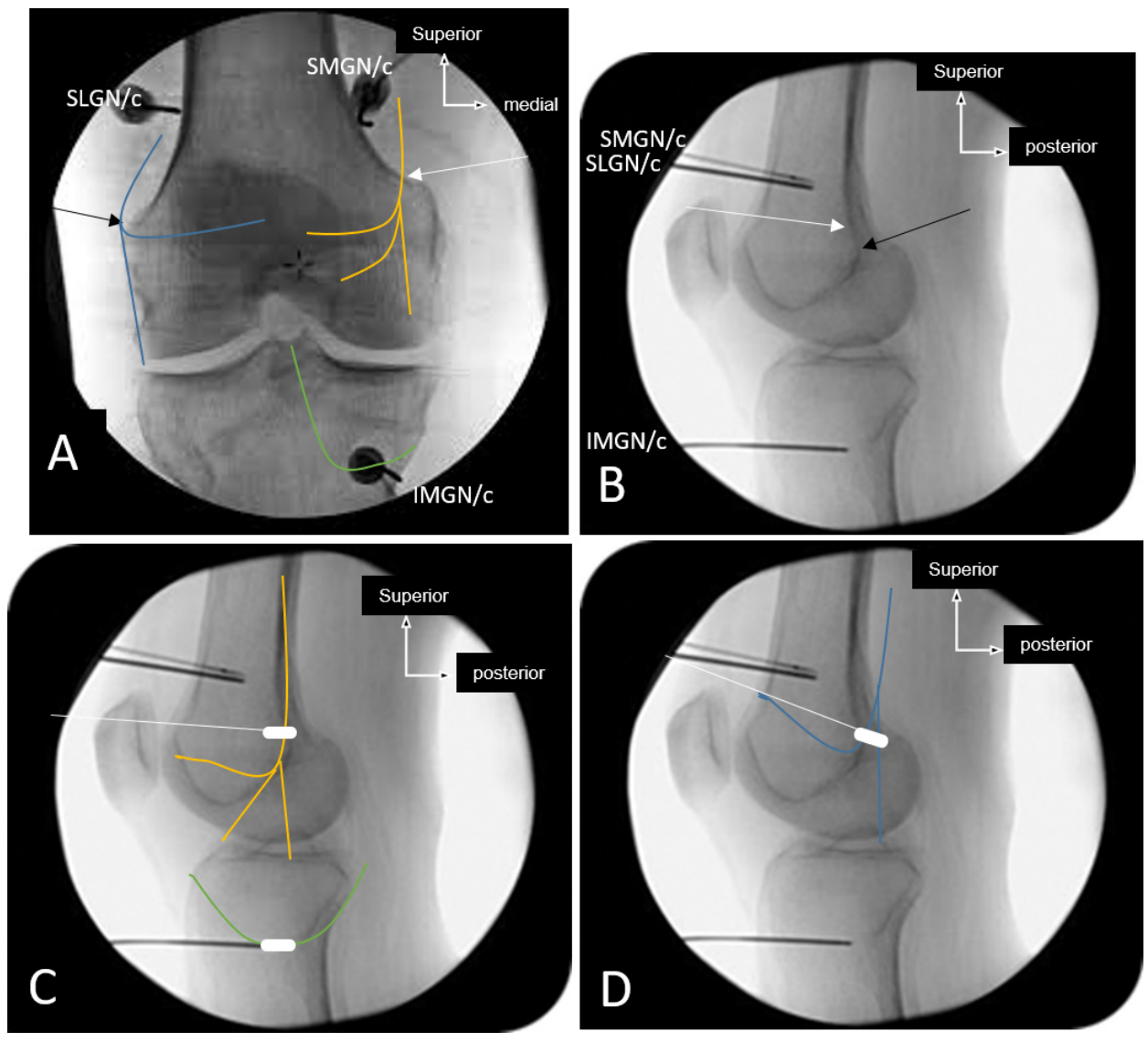

Figure 9 Differences between classical landmarks (SLGN/C, SMGN/C, and IMGN/c) and our proposed landmarks for fluoroscopic-guided GNB and RFA; the trajectory of the SLGN (blue) from sciatic nerve, SMGN (yellow) from nerve to vastus medialis and IMGN (green) from posterior articular nerve (branch of the sciatic nerve) have been drawn on classical fluoroscopic images. (A) A-P view and (B) lateral view. Classical fluoroscopic image of the final needle position during GNB/RFA. Note the important differences with our proposed treatment targets areas for the SMGN (white arrow) and SLGN (black arrow) on the A-P and lateral views. (C) Trajectory of the SMGN (yellow) and IMGN (green) intercepted by the active tip of the electrodes with our new positioning (white). Note that the classical electrode position (upper black electrode) could clearly not intercept the trajectory of SMGN. (D) SLGN trajectory (blue) intercepted by the active tip of the electrode with our new positioning (white). Note that the classical electrode position (upper black electrode) could clearly not intercept the trajectory of SLGN. A-P, anterior-posterior; GNB, genicular nerve block; IMGN, inferiormedial genicular nerve; IPBSN, infrapatellar branch of saphenous nerve; RFA, radiofrequency ablation; RFN, recurrent fibular nerve; SLGN, superior lateral genicular nerve; SMGN, superiormedial genicular nerve.

tubercle, deeply on the periosteum, provided $100 \%$ accuracy without any diffusion to the CFN.

The inferior-medial quadrant of the knee joint is innervated by the IPBSN and IMGN. We found that the classical landmarks used for needle placement to treat the IMGN were 100\% accurate, although this nerve was found to be mostly a branch of posterior articular nerve (from sciatic nerve) rather than tibial nerve like previously described. ${ }^{4} 18$ Concerning the IPBSN, its contribution to the sensory innervation of the inferior-medial region of the knee joint makes it an important therapeutic target. ${ }^{16} 17$ Contrary to Tran et al who reported that the IPBSN was mainly cutaneous and only innervated the inferior-medial quadrant of the anterior knee joint capsule in 3/15 (20\%) specimens, we and other authors ${ }^{23} 24$ found that the IPBSN supplied the inferior medial aspect of the anterior knee capsule and the overlying skin and subcutaneous tissue, in all the specimens. Its importance is reinforced by recent clinical studies where ablative treatment of IPBSN alone resulted in a significant decrease of knee pain in patients with knee OA, ${ }^{25-27}$ with results comparable with those observed in studies performing classical ablative therapy of three genicular nerve. ${ }^{4121322} \mathrm{Hu}$ et al ${ }^{20}$ demonstrated that a vertical treatment line originating at $50 \mathrm{~mm}$ medial to the apex patellae and connecting to a point just inferior to the level of tibial tuberosity would encompass $80 \%$ of the nerve innervating the anteromedial region of the inferior knee. Our landmark, adapted from $\mathrm{Hu}$ et al with a slight modification for more precision, was accurate in $100 \%$ of our specimens. Ablative therapy combining all these nerves, with more accurate treatment targets, would likely potentiate their analgesic effects. RFA therapy on the IPBSN should be applied deep enough to prevent cutaneous lesions. ${ }^{17}$

The teaching images in a recent article by Cushman et $a l^{28}$ suggested that, using Choi et al's ${ }^{4}$ targets, the SLGN and IMGN would likely be anesthetized and lesioned, whereas the SMGN would likely be anesthetized by $1.0 \mathrm{~mL}$ of local anesthetic, but may not be lesioned. In contrast, the current study (figure 9) found that the SMGN and SLGN would not be anesthetized or lesioned, while the IMGN would be using Choi et al's targets. The fundamental difference between the two studies is that we dissected all the limbs to visually check for accuracy that is, is the nerve dyed or not, while in their study, accuracy was hypothetically assessed using estimated courses of the nerves based on Tran et al's ${ }^{18}$ study. Since Cushman et al demonstrated that local injection of $0.5 \mathrm{~mL}$ of anesthetic spread beyond the boundaries of typical RFA lesions, the volume of anesthetic to be injected for accuracy studies should not be more than $0.5 \mathrm{~mL}$. Of course, 
a larger volume of anesthetic would spread on a greater surface (just $1 \mathrm{~mL}$ covers the entire A-P surface of the femur), ${ }^{28}$ and then, would capture all the nerves without any specificity, even if the needle placement is inaccurate. This may explain the higher rate of false positive diagnostic blocks. When analyzing the figure 3 provided by Cushman et $a l,{ }^{28}$ the SMGN would likely not be anesthetized by $0.5 \mathrm{~mL}$ of local anesthetic, nor be lesioned, just as we stated. Concerning the SLGN, the estimated course of the nerve depicted by Cushman $e a^{28}$ is not the same as we found from our dissections, nor the same as in figure $2 \mathrm{~b}$ provided by Tran $e t a l^{18}$ or figure 1 provided by Sutaria et al. ${ }^{19}$ The main trunk of the SLGN remains posterior (running with the biceps femoris) until it reaches the area connecting the posterior edge of the lateral surface of the femur to the lateral femoral condyle (figure 9) where it divides in its terminal branches. The Choi et al's targets could inconsistently capture the $\mathrm{LRN}^{19}$ or the NVL, ${ }^{7}$ but not the SLGN.

To date, the fluoroscopic-guided method is the most frequently used for GNB and RFA in the studies around the world. ${ }^{59}$ Since the targeted genicular nerves establish periosteal contact at the same anatomical areas before distributing to the knee joint, accurate needle placement can reliably be obtained by targeting bony landmarks under fluoroscopic guidance. ${ }^{47}$ This is a simple, accurate, and easily reproducible method. Its most important disadvantage is patient and physician exposure to radiation during the process. The correct superposition of both femoral condyles on the true lateral fluoroscopic image is a key of the success for accurate needle placement for SLGN and SMGN. Although the ultrasound-guided technique can provide similar results without radiation, it relies much more on equipment quality and operator-dependent skills. ${ }^{10}{ }^{29}$ In addition, the very small size of genicular nerves may not allow their direct visualization during ultrasonography ${ }^{529}$; the described procedure tends to visualize neurovascular bundle based on artery beat. ${ }^{29} 30$

The limitations of our study include the relatively small sample size. However, due to the consistent anatomical location of the distal trajectory of targeted genicular nerve despite some variation in their proximal trajectory, we postulate that these landmarks would follow the same patterns in most individuals. The proposed GNB/RFA technique only targets five nerves, not all the articular branches innervating the knee joint. Our study warrants further clinical trials to investigate the therapeutic benefit and safety of this revised technique compared with Choi's technique for fluoroscopic-guided GNB and RFA.

\section{CONCLUSION}

This study confirms that the anatomical landmarks currently used for fluoroscopy-guided GNB need to be revised for more accuracy. The findings of this study suggest that our updated anatomical landmarks for the five consistent articular nerves of the knee joint capsule (SMGN, IMGN, SLGN, RFN, and IPBSN) are accurate, safe and reproducible. Our study facilitates proper identification and needle placement during GNB procedures with fluoroscopic guidance. These findings provide physicians with a better technique to accurately target a larger panel of consistent genicular nerves for selective denervation, which could potentially lead to improved treatment for patients suffering from chronic knee pain.

Contributors $\mathrm{LF}, \mathrm{CB}$ and $\mathrm{OC}$ contributed to the conception and design. $\mathrm{LF}$ and $\mathrm{CB}$ contributed to the cadaveric dissections, data collection and analysis. AS and BLPDW contributed to the data analysis. JEKK provided assistance in cadaveric dissections. LF contributed to the manuscript writing. AS, BLPDW, JEKK, CB and OC contributed to the manuscript revision. $\mathrm{OC}$ is the project manager.
Funding The authors have not declared a specific grant for this research from any funding agency in the public, commercial or not-for-profit sectors.

Competing interests None declared.

Patient consent for publication Not required.

Ethics approval Ethics approval was received from the Research Ethic Board for health sciences of the Université Catholique de Louvain.

Provenance and peer review Not commissioned; externally peer reviewed.

\section{REFERENCES}

1 Crawford DC, Miller LE, Block JE. Conservative management of symptomatic knee osteoarthritis: a flawed strategy? Orthop Rev 2013;5:e2.

2 Wylde V, Hewlett S, Learmonth ID, et al. Persistent pain after joint replacement: prevalence, sensory qualities, and postoperative determinants. Pain 2011;152:566-72

3 Lavand'homme P, Thienpont E. Pain after total knee arthroplasty: a narrative review focusing on the stratification of patients at risk for persistent pain. Bone Joint $J$ 2015;97-B(10 Suppl A):45-8.

4 Choi W-J, Hwang S-J, Song J-G, et al. Radiofrequency treatment relieves chronic knee osteoarthritis pain: a double-blind randomized controlled trial. Pain 2011;152:481-7.

5 Yasar E, Kesikburun S, Kılıç C, et al. Accuracy of ultrasound-guided Genicular nerve block: a cadaveric study. Pain Physician 2015;18:E899-904.

6 Kim D-H, Choi S-S, Yoon S-H, et al. Ultrasound-Guided Genicular nerve block for knee osteoarthritis: a double-blind, randomized controlled trial of local anesthetic alone or in combination with corticosteroid. Pain Physician 2018;21:41-52.

7 Franco CD, Buvanendran A, Petersohn JD, et al. Innervation of the anterior capsule of the human knee: implications for radiofrequency ablation. Reg Anesth Pain Med 2015:40:363-8.

8 Kim SY, Le PU, Kosharskyy B, et al. Is Genicular nerve radiofrequency ablation safe? A literature review and anatomical study. Pain Physician 2016;19:E697-705.

9 El-Hakeim EH, Elawamy A, Kamel EZ, et al. Fluoroscopic guided radiofrequency of Genicular nerves for pain alleviation in chronic knee osteoarthritis: a single-blind randomized controlled trial. Pain Physician 2018;21:169-77.

10 Hernández-González L, Calvo CE, Atkins-González D. Peripheral nerve radiofrequency neurotomy: hip and knee joints. Phys Med Rehabil Clin NAm 2018;29:61-71.

11 Jamison DE, Cohen SP. Radiofrequency techniques to treat chronic knee pain: a comprehensive review of anatomy, effectiveness, treatment parameters, and patient selection. J Pain Res 2018;11:1879-88.

12 Qudsi-Sinclair S, Borrás-Rubio E, Abellan-Guillén JF, et al. A comparison of Genicular nerve treatment using either radiofrequency or analgesic block with corticosteroid for pain after a total knee arthroplasty: a double-blind, randomized clinical study. Pain Pract 2017;17:578-88.

13 McCormick ZL, Korn M, Reddy R, et al. Cooled radiofrequency ablation of the Genicular nerves for chronic pain due to knee osteoarthritis: six-month outcomes. 2017;18:1631-41.

14 McCormick ZL, Reddy R, Korn M, et al. A prospective randomized trial of prognostic Genicular nerve blocks to determine the predictive value for the outcome of cooled radiofrequency ablation for chronic knee pain due to osteoarthritis. 2018;19:1628-38.

15 Bendtsen TF, Moriggl B, Chan V, et al. The optimal analgesic block for total knee arthroplasty. Reg Anesth Pain Med 2016;41:711-9.

16 Burckett-St Laurant D, Peng P, Girón Arango L, et al. The nerves of the adductor canal and the innervation of the knee: an anatomic study. Reg Anesth Pain Med 2016:41:321-7.

17 Orduña Valls JM, Vallejo R, López Pais P, et al. Anatomic and ultrasonographic evaluation of the knee sensory innervation: a cadaveric study to determine anatomic targets in the treatment of chronic knee pain. Reg Anesth Pain Med 2017:42:90-8.

18 Tran J, Peng PWH, Lam K, et al. Anatomical study of the innervation of anterior knee joint capsule: implication for image-guided intervention. Reg Anesth Pain Med 2018;43:407-14

19 Sutaria RG, Lee SW, Kim SY, et al. Localization of the lateral Retinacular nerve for diagnostic and therapeutic nerve block for lateral knee pain: a cadaveric study. $P m R$ 2017:9:149-53.

20 Hu E, Preciado J, Dasa V, et al. Development and validation of a new method for locating patella sensory nerves for the treatment of inferior and superior knee pain. $J$ Exp Orthop 2015;2

21 Fonkoué L, Behets C, Kouassi Jean-Éric K, et al. Distribution of sensory nerves supplying the knee joint capsule and implications for genicular blockade and radiofrequency ablation: an anatomical study. Surg Radiol Anat. In Press 2019. doi:10.1007/s00276-019-02291-y. [Epub ahead of print: 23 Jul 2019].

22 Bhatia A, Peng P, Cohen SP. Radiofrequency procedures to relieve chronic knee pain an evidence-based narrative review. Reg Anesth Pain Med 2016:41:501-10.

23 Hirasawa Y, Okajima S, Ohta M, et al. Nerve distribution to the human knee joint: anatomical and immunohistochemical study. Int Orthop 2000:24:1-4.

24 Horner G, Dellon AL. Innervation of the human knee joint and implications for surgery. Clin Orthop Relat Res 1994;(301):221-6. 
25 Akbas M, Luleci N, Dere K, et al. Efficacy of pulsed radiofrequency treatment on the saphenous nerve in patients with chronic knee pain. J Back Musculoskelet Rehabil 2011;24:77-82.

26 Radnovich R, Scott D, Patel AT, et al. Cryoneurolysis to treat the pain and symptoms of knee osteoarthritis: a multicenter, randomized, double-blind, sham-controlled trial. Osteoarthritis Cartilage 2017;25:1247-56.

27 Baysal PK, Baysal Özgür, Erkllinç A, et al. Is saphenous nerve radio frequency an effective treatment for advanced gonarthrosis in elders with cardiac comorbidity? J Back Musculoskelet Rehabil 2018;31:113-8.
28 Cushman DM, Monson N, Conger A, et al. Use of $0.5 \mathrm{~mL}$ and $1.0 \mathrm{~mL}$ of local anesthetic for Genicular nerve blocks. Pain Med 2019;20:1049-52.

29 Sarı S, Aydın ON, Turan Y, et al. Which imaging method should be used for genicular nerve radio frequency thermocoagulation in chronic knee osteoarthritis? J Clin Monit Comput 2017;31:797-803.

30 González Sotelo V, Maculé F, Minguell J, et al. Ultrasound-Guided genicular nerve block for pain control after total knee replacement: preliminary case series and technical note. Rev Esp Anestesiol Reanim 2017;64:568-76. 